\title{
Use of Deep-Learning Genomics to Discriminate Healthy Individuals from Those with Alzheimer's Disease or Mild Cognitive Impairment
}

\author{
Lanlan Li, ${ }^{1}$ Yeying Yang, ${ }^{2}$ Qi Zhang, ${ }^{1}$ Jiao Wang, ${ }^{3}$ Jiehui Jiang $\mathbb{D}^{1}$ \\ and Alzheimer's Disease Neuroimaging Initiative ${ }^{4}$ \\ ${ }^{1}$ Institute of Biomedical Engineering, School of Communication and Information Engineering, Shanghai University, \\ Shanghai 200444, China \\ ${ }^{2}$ LongHua Hospital, Shanghai University of Traditional Chinese Medicine, Shanghai 200032, China \\ ${ }^{3}$ School of Life Science, Shanghai University, Shanghai 200444, China \\ ${ }^{4}$ Indiana University School of Medicine, Indianapolis, IN 46202, USA
}

Correspondence should be addressed to Jiehui Jiang; jiangjiehui@shu.edu.cn

Received 14 May 2021; Accepted 11 June 2021; Published 15 July 2021

Academic Editor: Muh-Shi Lin

Copyright ( 92021 Lanlan Li et al. This is an open access article distributed under the Creative Commons Attribution License, which permits unrestricted use, distribution, and reproduction in any medium, provided the original work is properly cited.

Objectives. Alzheimer's disease (AD) is the most prevalent neurodegenerative disorder and the most common form of dementia in the elderly. Certain genes have been identified as important clinical risk factors for $\mathrm{AD}$, and technological advances in genomic research, such as genome-wide association studies (GWAS), allow for analysis of polymorphisms and have been widely applied to studies of AD. However, shortcomings of GWAS include sensitivity to sample size and hereditary deletions, which result in low classification and predictive accuracy. Therefore, this paper proposes a novel deep-learning genomics approach and applies it to multitasking classification of $\mathrm{AD}$ progression, with the goal of identifying novel genetic biomarkers overlooked by traditional GWAS analysis. Methods. In this study, we selected genotype data from 1461 subjects enrolled in the Alzheimer's Disease Neuroimaging Initiative, including 622 AD, 473 mild cognitive impairment (MCI), and 366 healthy control (HC) subjects. The proposed deep-learning genomics (DLG) approach consists of three steps: quality control, coding of singlenucleotide polymorphisms, and classification. The ResNet framework was used for the DLG model, and the results were compared with classifications by simple convolutional neural network structure. All data were randomly assigned to one training/validation group and one test group at a ratio of 9:1. And fivefold cross-validation was used. Results. We compared classification results from the DLG model to those from traditional GWAS analysis among the three groups. For the AD and HC groups, the accuracy, sensitivity, and specificity of classification were, respectively, $98.78 \pm 1.50 \%, 98.39 \% \pm 2.50 \%$, and $99.44 \% \pm 1.11 \%$ using the DLG model, while $71.38 \% \pm 0.63 \%, 63.13 \% \pm 2.87 \%$, and $85.59 \% \pm 6.66 \%$ using traditional GWAS. Similar results were obtained from the other two intergroup classifications. Conclusion. The DLG model can achieve higher accuracy and sensitivity when applied to progression of AD. More importantly, we discovered several novel genetic biomarkers of AD progression, including rs6311 and rs6313 in HTR2A, rs1354269 in NAV2, and rs690705 in RFC3. The roles of these novel loci in $\mathrm{AD}$ should be explored in future research. 


\section{Introduction}

Alzheimer's disease (AD) is the most common type of dementia and is an irreversible, progressive neurological brain disorder typically beginning with mild memory decline; in time, it can seriously impair an individual's ability to carry out daily activities and lead to loss of autonomy [1, 2]. Mild cognitive impairment (MCI) is a preclinical stage of $\mathrm{AD}$, in which individuals have no obvious cognitive behavioral symptoms but can show subtle prodromal signs of dementia $[3,4]$. It is widely recognized that early detection of $\mathrm{AD}$ and $\mathrm{MCI}$ is essential to slowing progression.

Among factors that influence AD progression, common genetic variants are major risk factors [5]. Currently, the development of cheap comprehensive genetic testing of peripheral blood has brought dramatic changes to studies of the mechanisms of disease development. In recent decades, several genes have been associated with $\mathrm{AD}$ risk based on full-genome genotyping arrays using blood samples $[6,7]$. For instance, genomics analysis showed APOE to be the most strongly associated $\mathrm{AD}$ risk gene [8]. In addition, the CLU, PICALM, SORL1, BIN1, and TOMM40 genes have also been identified as $\mathrm{AD}$ risk factors in the literature [7, 9, 10].

Technological advances [11] have allowed analysis of millions of nucleotide polymorphisms from thousands of subjects, including advanced genome-wide association studies (GWAS) and whole genome sequencing [12-16] that have increased our understanding of the genetic complexity of AD susceptibility. For instance, recent GWAS from the Alzheimer's Disease Neuroimaging Initiative (ADNI) have related known $\mathrm{AD}$ risk genes to differences in rates of brain atrophy and biomarkers of $\mathrm{AD}$ in the cerebrospinal fluid [17]. Moreover, the International Genomics of Alzheimer's Project studied 74046 participants, confirming nearly all of the previous genetic risk factors and identifying 12 new susceptibility loci for AD [18]. Therefore, genomics analysis, especially GWAS analysis, has yielded important advances in $\mathrm{AD}$ research.

However, there are some limitations of GWAS. Firstly, traditional GWAS intergroup analysis is distorted by differences in sample sizes [19]. Secondly, traditional GWAS analysis is strongly dependent on prior knowledge and hand coding, which requires much time and energy and risks bias or errors in data entry [16] that can result in poor repeatability. Moreover, although traditional GWAS analysis can assure high specificity of disease screening, accuracy, and sensitivity are relatively low. In practice, false positives are preferred over false negatives in order to avoid omissions in disease screening. Therefore, alternative analytical tools would help to drive novel hypotheses and models.

Deep-learning algorithms implemented via deep neural networks can automatically embed computational features to yield end-to-end models that facilitate discovery of relevant highly complex features [20]. Seminal studies in 2015 demonstrated the applicability of deep neural networks to DNA sequence data $[21,22]$. Deep convolutional neural networks (CNNs) have been used in recent studies to predict various molecular phenotypes on the basis of DNA sequence alone. Applications include classifying transcription factor binding sites, predicting molecular phenotypes such as DNA methylation, microRNA targets, and gene expression [23-27]. In addition, CNNs have been utilized to call genetic variants [28] and classify genetic mutations in tumors [29]. Multitask and multimodal models and transfer learning have also been developed in genomics $[30,31]$. In this work, we hypothesize that deep-learning genomics (DLG) can be applied to AD and outperform traditional GWAS analysis. We propose a DLG method to replace traditional GWAS analysis for multitasking classification of $\mathrm{AD}$ progression and use this approach to seek novel genetic biomarkers of AD susceptibility.

\section{Materials and Methods}

The experimental workflow of this study consisted of three steps as shown in Figure 1. First, we conducted quality control and SNP genotype coding for SNP genotype data. Second, we used the deep residual network ResNet for DLG. The goal of the deep residual network was to obtain a model by supervised learning for prediction and extraction of DLG features. The details of this process are described in detail in the following sections. Finally, we investigated interpretability of the DLG model by applying Gradient-weighted Class Activation Mapping (Grad-CAM).

2.1. Subjects. Data used in the preparation of this study was obtained from the ADNI database (http://adni.loni.usc.edu/ ). ADNI was launched in 2004 by the National Institute on Aging, the National Institute of Biomedical Imaging and Bioengineering, the Food and Drug Administration, private pharmaceutical companies, and nonprofit organizations, as a \$60 million, 5-year public-private partnership. In this study, 1461 individuals (622 AD, $473 \mathrm{MCI}$, and 366 healthy controls (HCs)) from the ADNI 1, ADNI 2, and ADNI GO cohorts of the ADNI database were included. Meanwhile, the following data from the 1461 ADNI participants was downloaded: Illumina SNP genotyping data, demographic information, and diagnosis information. Written informed consent was obtained from all participants, and the study was conducted with prior institutional review board approval. Clinical characteristics, including age, sex, education, and Montreal Cognitive Assessment (MoCA) results, were collected and are listed in Table 1.

The subjects were of age 55-90 (inclusive) years. The detailed ADNI eligibility criteria are available from http:// adni.loni.usc.edu/methods/documents/. In brief, eligibility criteria for these participants were as follows: (1) normal subjects: a Clinical Dementia Rating (CDR) of 0, nondepressed, non-MCI, and nondemented; (2) MCI subjects: a memory complaint, objective memory loss measured by education adjusted scores on Wechsler Memory Scale 7/Logical Memory II, a CDR of 0.5 , absence of significant levels of impairment in other cognitive domains, essentially preserved activities of daily living, and an absence of dementia; (3) AD: CDR of 0.5 or 1.0 and met the National Institute of Neurological and Communicative Disorders and Stroke and Alzheimer's disease and Related Disorders Association 


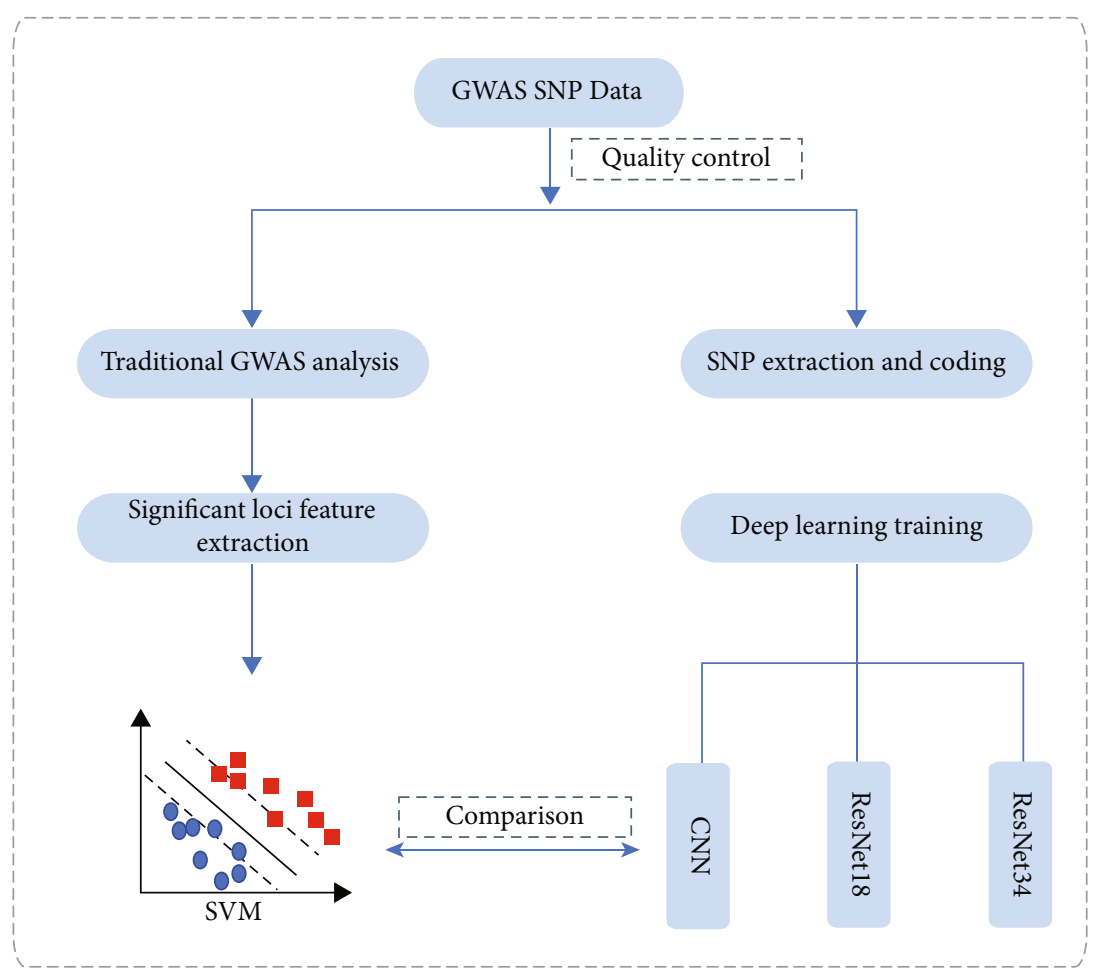

Figure 1: The flowchart of experimental procedures in this study.

TABLE 1: Clinical and baseline demographic characteristics of all participants.

\begin{tabular}{|c|c|c|c|c|}
\hline Groups & Gender (M/F) & Age (years) & Education & MoCA \\
\hline \multicolumn{5}{|c|}{ Training and validation group $(n=1316)$} \\
\hline $\mathrm{AD}(n=560)$ & $320 / 240^{\mathrm{a}}$ & $74.42 \pm 7.26$ & $15.54 \pm 2.85^{\mathrm{a}}$ & $17.18 \pm 5.05^{\mathrm{a}, \mathrm{b}}$ \\
\hline MCI $(n=426)$ & $255 / 171^{\mathrm{c}}$ & $73.27 \pm 7.39$ & $15.98 \pm 2.78^{\mathrm{c}}$ & $23.62 \pm 2.95^{\mathrm{b}, \mathrm{c}}$ \\
\hline $\mathrm{HC}(n=330)$ & $163 / 167^{\mathrm{a}, \mathrm{c}}$ & $73.80 \pm 5.84$ & $16.46 \pm 2.54^{\mathrm{a}, \mathrm{c}}$ & $25.88 \pm 2.42^{\mathrm{a}, \mathrm{c}}$ \\
\hline \multicolumn{5}{|c|}{ Test group $(n=145)$} \\
\hline $\mathrm{AD}(n=62)$ & $41 / 21^{\mathrm{a}}$ & $75.71 \pm 7.99$ & $15.55 \pm 3.32$ & $13.91 \pm 6.82^{\mathrm{b}}$ \\
\hline MCI $(n=47)$ & $30 / 17^{\mathrm{c}}$ & $75.56 \pm 7.94$ & $14.81 \pm 3.70$ & $22.75 \pm 3.31^{b}$ \\
\hline $\mathrm{HC}(n=36)$ & $14 / 22^{\mathrm{a}, \mathrm{c}}$ & $75.45 \pm 3.49$ & $15.58 \pm 3.59$ & - \\
\hline
\end{tabular}

Data of age and education were presented as mean \pm standard deviation. MoCA: Montreal Cognitive Assessment. Group-level two-sample $t$ test is conducted for age, education, and MoCA. Group-level chi-square test is conducted for gender. ${ }^{\mathrm{a}} p$ value $\mathrm{HC}$ vs. $\mathrm{AD} ;{ }^{\mathrm{b}} p$ value $\mathrm{AD}$ vs. MCI; ${ }^{\mathrm{c}} p$ value $\mathrm{HC}$ vs. MCI.

criteria for probable AD [32]. Specific psychoactive medications were excluded.

We investigated two groups of subjects using SNP genotype data collected from the ADNI databases. Our training and validation group contained 560 subjects with $\mathrm{AD}, 426$ subjects with $\mathrm{MCI}$, and $330 \mathrm{HC}$ subjects. We used the SNP genotype data from this group to establish and test the validity of our predictive models. Our test group consisted of 62 $\mathrm{AD}$ subjects, $47 \mathrm{MCI}$ subjects, and $36 \mathrm{HC}$ controls, and we used the SNP genotype data to evaluate the diagnostic value of the predictive models.

2.2. DNA Isolation and SNP Genotyping. SNP genotyping for more than 620,000 target SNPs was completed on all ADNI participants using the following protocol. First, a total of 7 $\mathrm{mL}$ of blood was taken from each participant and stored in EDTA-containing Vacutainer tubes, and genomic DNA was extracted using the QIAamp DNA Blood Maxi Kit following the manufacturer's protocol. Second, lymphoblastoid cell lines were established by transforming B lymphocytes with Epstein-Barr virus [33]. Fourteen genomic DNA samples were analyzed using the Human 610-Quad BeadChip according to the manufacturer's protocols. Before starting the assay, a $50 \mathrm{ng}$ sample of genomic DNA from each participant was examined qualitatively on a $1 \%$ Tris-acetate-EDTA agarose gel to check for degradation. Degraded DNA samples were excluded from further analysis. Third, samples were quantitated in triplicate with PicoGreen ${ }^{\circledR}$ reagent and diluted 
to $50 \mathrm{ng} / \mathrm{L}$ in TrisEDTA buffer $(10 \mathrm{mM}$ Tris, $1 \mathrm{mM}$ EDTA, $\mathrm{pH}$ 8.0). A total of $200 \mathrm{ng}$ of DNA was denatured, neutralized, and amplified for 22 hours at $37^{\circ} \mathrm{C}$, and then fragmented with FMS reagent (Illumina) at $37^{\circ} \mathrm{C}$ for 1 hour, precipitated with 2-propanol, and incubated at $4^{\circ} \mathrm{C}$ for 30 minutes. Fourth, the resulting blue precipitate was resuspended in RA1 reagent (Illumina) at $48^{\circ} \mathrm{C}$ for 1 hour. Samples were then denatured $\left(95^{\circ} \mathrm{C}\right.$ for 20 minutes) and immediately hybridized onto BeadChips at $48^{\circ} \mathrm{C}$ for 20 hours. The BeadChips were washed and subjected to single base extension and staining. Finally, the BeadChips were coated with XC4 reagent (Illumina), desiccated, and imaged on a BeadArray Reader (Illumina). Illumina BeadStudio 3.2 software was used to generate SNP genotypes from bead intensity data.

2.3. Quality Control and APOE Genotype. The following quality control (QC) steps were performed on the 1461 samples using PLINK v1.07 software. QC processes were conducted separately between the $\mathrm{AD}$ and $\mathrm{HC}$ groups, the $\mathrm{HC}$ and MCI groups, and the AD and MCI groups. SNPs and participants were excluded from the analysis if they failed to meet any of the following criteria [34]: call rate per SNP $\geq 90 \%$; call rate per participant $\geq 90 \%$; gender check; minor allele frequency $(\mathrm{MAF}) \geq 5 \%$; Hardy-Weinberg equilibrium test of $p \leq 10^{-6}$; PI_HAT $<0.5$. After the QC procedure, the numbers of features considered for future analysis of each subject in the paired groups were as follows: 301,388 in the $\mathrm{HC}$ and MCI groups, 301,853 in the HC and MCI groups, and 301,138 in the MCI and AD groups. The overall genotyping rate for the remaining dataset was over $99.5 \%$.

In addition, although the APOE gene is an important target gene in $\mathrm{AD}$ research, it was not available for all identified APOE SNPs on the Illumina array. Therefore, based on the reported APOE $\varepsilon 2 / \varepsilon 3 / \varepsilon 4$ status, the genotypes of the unavailable APOE SNPs were added manually to ADNI genotype data before assessing sample quality.

2.4. SNP Genotype Coding. A single-nucleotide polymorphism is a DNA sequence variation which occurs when a single nucleotide (A, T, C, or G) in the genome differs among members of a biological species or across paired chromosomes. Based on the satisfactory ADNI GWAS SNP data of this study, we encoded SNPs using the following coding scheme: 1 refers to A, 2 refers to $\mathrm{T}, 3$ refers to $\mathrm{C}$, and 4 refers to $\mathrm{G}$.

2.5. GWAS Analysis. In the multitasking classification of this study, GWAS analysis, which has emerged as a popular tool for identifying genetic variants associated with disease risk, was designed to be compared with deep-learning models. Standard analysis of a case-control GWAS involves assessing the association between each individual genotyped SNP and disease risk. Manhattan and quantile-quantile $(Q-Q)$ plots were used to visualize the GWAS results. All association results surviving the significance threshold of $p<1.66 e^{-7}$ were saved and prepared for subsequent pattern analysis.

2.6. Deep-Learning Genomics Model Based on ResNet. The DLG model acted as a feature encoder, which had a significant impact on classification. In this study, we applied
ResNet, a deep residual network, to the classification between $\mathrm{AD}$ and $\mathrm{HC}$ groups, $\mathrm{AD}$ and MCI groups, and $\mathrm{HC}$ and $\mathrm{MCI}$ groups. Residual units were added to the deep residual network on the basis of CNNs.

A CNN, the most effective type deep-learning model, is generally composed of three types of layers: convolutional, pooling, and fully connected. The following describes the operation of a CNN. The first step is to convolve the input sequences with a set of filter kernels; all the features active at different positions after convolution constitute the feature map [35]. A nonlinear activation function, typically a rectified linear unit (ReLU), is applied on each layer and on the sum of the feature maps. The operation of the convolutional layer and ReLU can be expressed as follows:

$$
\begin{gathered}
C_{n}^{r}=\operatorname{ReLU}\left(\sum_{m} v_{m}^{r-1} * w_{n}^{r}+b_{n}^{r}\right), \\
\left\{\begin{array}{l}
\operatorname{ReLU}\left(y_{n}\right)=\max \left(0, y_{n}\right), \\
y_{n}=\sum_{m} v_{m}^{r-1} * w_{n}^{r}+b_{n}^{r},
\end{array}\right.
\end{gathered}
$$

where $C_{n}^{r}$ is the $n^{\text {th }}$ output of the $r^{\text {th }}$ convolutional layer, $n$ represents the number of filters in the $r^{\text {th }}$ layer, $w_{n}^{r}$ and $b_{n}^{r}$ are, respectively, the weight and bias of the $n^{\text {th }}$ filter of the $r^{\text {th }}$ layer, $v_{m}^{r-1}$ is the $m^{\text {th }}$ output of previous layer $r-1$, and * denotes the convolutional operation.

Next, the resulting feature map is processed through the pooling layer by taking either the mean or maximum activation over disjoint regions for each channel $[20,35]$. By sequential combination of convolutional and pooling layers, a multilayer structure is built for feature description. Lastly, the fully connected layers are employed for classification. In total, when given a training set $\left\{X_{j}\right\}_{j}$, the learning process of a CNN with $K$ convolutional layers, whose filter parameters are $\left\{W_{i}\right\}_{i=1}^{K}$, the bias values are $\left\{b_{i}\right\}_{i=1}^{K}$, and $D$ refers to classification layers, can be represented as an optimization learning task:

$$
\min _{\left\{W_{i}\right\}_{i=1}^{K},\left\{b_{i}\right\}_{i=1}^{K}} \sum_{j} L\left(h\left(X_{j}\right), f\left(\left\{W_{i}\right\}_{i=1}^{K},\left\{b_{i}\right\}_{i=1}^{K}, D\right)\right),
$$

where $L$ is the loss function that represents the cost difference between the true label $h(X)$ and the predictive label from the CNN model $f\left(X,\left\{W_{i}\right\}_{i=1}^{K},\left\{b_{i}\right\}_{i=1}^{K}, D\right)$.

Based on the CNN model, the greatest advantage of the ResNet framework lies in adding identity mapping that is performed by the shortcut connections, the outputs of which are added to the outputs of the stacked layers [36]. Therefore, the ResNet addressed the degradation problem and added neither extra parameters nor computational complexity. The formula for residual learning was designed as follows: the desired underlying mapping is denoted as $H(x)$, and the stacked nonlinear layers were allowed to fit a separate mapping of $F(x, \Theta)=H(x)-x$. The original mapping was recast into $F(x, \Theta)+x$. Thus, the overall representation of the residual block was as follows: 


$$
H(x)=\mathrm{F}(x, \Theta)+x
$$

The formulation of $\mathrm{F}(x, \Theta)+x$ can be realized by feedforward neural networks using "shortcut connections." A deep residual network can be established by stacking a series of residual blocks. Specifically, there were two steps in the process: forward computation and backward propagation. When $K$ residual blocks are chosen to stack, the forward propagation of such a structure can be expressed by

$$
x_{K}=x_{0}+\sum_{r=1}^{K} \mathrm{~F}\left(x_{r-1}, \Theta_{r-1}\right) \text {, }
$$

where $x_{0}$ and $x_{1}$ are the input and the output of the residual network, respectively, and $\Theta_{r}=\left\{\left.\theta_{r, l}\right|_{1 \leq l \leq L}\right\}$ is the weight related to the $r_{\text {th }}$ residual block, $L$ being the number of layers within the block.

Likewise, the back propagation of the overall loss of the neural network to $x_{0}$ can be denoted as

$$
\frac{\partial L}{\partial x_{0}}=\frac{\partial L}{\partial x_{K}}\left(1+\frac{\partial}{\partial x_{0}} \sum_{i=1}^{K} \mathrm{~F}\left(x_{i-1}, \Theta_{i-1}\right)\right),
$$

where $L$ is the whole loss function of the neural network.

Before modeling using the above procedures, each subject's SNP genotype data was cropped after quality control and mapped to $776 \times 776$ pixels. The pathology type was encoded to one-hot, which was the label. Thereafter, in the training stage, SNP genotype data was fed into the network to update model parameters via backward propagation with the Adam algorithm, a first-order gradient-based optimization algorithm which has been proven to be computationally efficient and appropriate for training deep neural networks. The outputs of the network were used as the classification results, and the crossentropy of the outputs was calculated as the loss function. More specifically, the output of the network for each individual SNP could be a binary value. 1 represented the highest probability of being $\mathrm{AD}$ subjects, while 0 represented highest probability of being HC subjects.

We adopted ResNet18 and ResNet34 frameworks in this study. Meanwhile, we also utilized a traditional CNN model for the comparative experiments of classification. In the ResNet models, we set learning rate into $1 e^{-3}$ and applied the Adam optimizer to update the model parameters with the batch size of 8 . The maximum number of iterations was set into 20. Note that we used L2 regularization in this step to prevent the overfit of our model. For adjusting the CNN model parameters, we set learning rate into $1 e^{-2}$ and applied the Adam optimizer to update the model parameters with the batch size of 8 . The maximum number of iterations was set to 30. Above deep-learning models were processed on a GPU (graphics processing unit, GTX $1080 \mathrm{Ti}$ acceleration of PyCharm 3.5).

For investigating the interpretability of the DLG model, the last convolutional layer of the last res-block was made transparent in order to extract DLG features by applying Grad-CAM and two-sample $t$-tests. For the first step, the last convolutional layer of the last res-block was chosen to extract normalized DLG features. Subsequently, using a two-sample $t$-test with a false discovery rate $[37,38]$, we compared the $Z$ -coefficients of the $\mathrm{AD}$ and $\mathrm{HC}$ groups, the $\mathrm{HC}$ and $\mathrm{MCI}$ groups, and the $\mathrm{MCI}$ and $\mathrm{AD}$ groups.

2.7. Classification. In this study, the subjects of multitasking classification were randomly divided into one training group and one independent test group at a ratio of $9: 1$ as shown in Table 1 . The training group was then used to optimize the model parameters. We also randomly chose $25 \%$ of training group to form a validation group to guide the choice of hyperparameters.

On the one hand, we conducted training of several deeplearning models, including ResNet18, ResNet34, and a traditional $\mathrm{CNN}$, and compared classification performance in order to screen for the optimum DLG. On the other hand, in order to verify the diagnostic capabilities of the DLG model compared with traditional GWAS analysis, we also designed comparative trials. Among all the gene indicators, theta proved to be the most directly related to SNP changes. APOE $\varepsilon 4$ status and the normalized theta-value of the significant SNP loci found in this study were seen to be genetic predictors, and we used the support vector machine (SVM) with the linear kernel 500 times for classification of traditional GWAS.

To evaluate classification performance, we repeatedly conducted 5 -fold crossvalidation in the training group. Accuracy, sensitivity, and specificity were used to evaluate the results. The mathematical expression of the three parameters was as follows:

$$
\begin{aligned}
\text { Accuracy } & =\frac{T n+T p}{T n+T p+F n+F p}, \\
\text { Sensitivity } & =\frac{T p}{T p+F n}, \\
\text { Specificity } & =\frac{T n}{T n+F p},
\end{aligned}
$$

where $T n, T p, F n$, and $F p$ denote, respectively, true negatives, true positives, false negatives, and false positives.

A receiver-operating characteristic (ROC) curve was produced to intuitively compare the results of the different approaches, and the area under the curve (AUC) of the ROC was computed to quantitatively evaluate classification performance.

2.8. Statistical Analysis. Demographic characteristics were compared between groups using a two-sample $t$-test or the chi-square test. In addition, a two-sample $t$-test of the extracted features was applied as a criterion to estimate the differences in DLG features between AD patients and HCs, $\mathrm{AD}$ patients and MCIs, and HCs and MCIs. All statistical analyses were performed using SPSS Version 22.0 software (SPSS Inc., Chicago, IL) and Matlab2016b (Mathworks Inc., Sherborn, MA, United States). All $p$ values $<0.05$ were considered significant. 


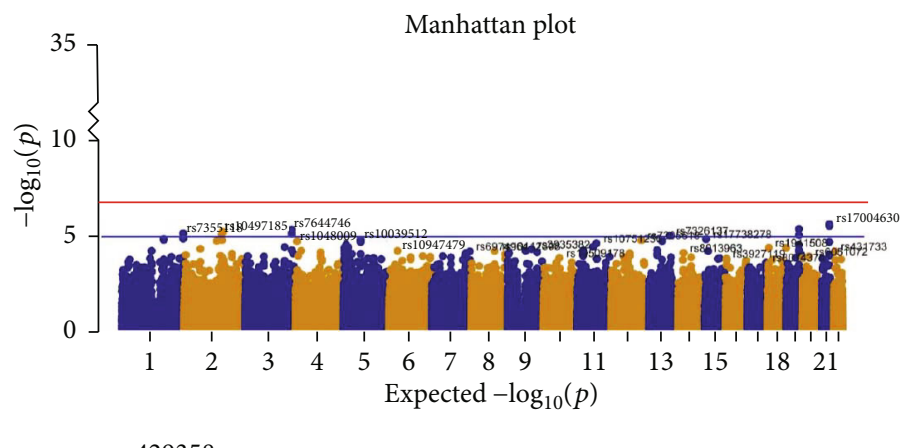

- rs429358

- rs2075650

FIGURE 2: Manhattan plot of genome-wide association study (GWAS) between AD and HC groups. The $y$-axis shows the $p$ value (on the $\log 10$ scale) for each association test. The $x$-axis is the chromosomal position of each SNP. The horizontal lines in the Manhattan plot display the cutoffs for two significant levels: blue line for $p<10^{-5}$ (generally significant level) and red line for $p<1.66 e^{-7}$.

\section{Results}

3.1. Outcomes of GWAS Analysis. We carried out casecontrol GWAS analysis between the $\mathrm{AD}$ and $\mathrm{HC}$ groups and observed two genome-wide significant loci on chromosome 19, including rs429358 (APOE, the epsilon 4 marker) and rs2075650 (TOMM40). Figures 2 and 3 show the resulting Manhattan and $Q-Q$ plots, and Table 2 summaries the SNPs that achieved genome-wide significance. The $p$ value used to assess significant differences was calculated as $p=$ $0.05 / N$, where $N$ indicates the number of satisfied SNPs.

3.2. Classification Performance. Table 3 lists the performance of the different multitasking classification methods, including classification accuracy, sensitivity, specificity, and AUC. Taking the result of classification between the $\mathrm{AD}$ and $\mathrm{HC}$ test group subjects as an example, accuracy, sensitivity, specificity, and AUC were, respectively, $71.38 \% \pm 0.63 \%, 63.13 \% \pm$ $2.87 \%, 85.59 \% \pm 6.66 \%$, and 0.744 , for the GWAS analysis, $92.45 \% \pm 8.13 \%, 93.87 \pm 12.26,90.00 \pm 15.97$, and 0.915 for the CNN model, $97.96 \pm 1.71,97.42 \pm 3.16,98.89 \pm 1.36$, and 0.980 for ResNet18, and $98.78 \% \pm 1.50 \%, 98.39 \% \pm$ $2.50 \%, 99.44 \% \pm 1.11 \%$, and 0.981 for ResNet34. We found that the deep-learning model exhibited high accuracy, sensitivity, and specificity, whereas accuracy and sensitivity were low for the GWAS analysis. Therefore, we concluded that deep-learning models were superior to traditional GWAS analysis for classification. And compared with the CNN model, the results using ResNet were more robust and stable. These results were the same using the other two group-level classifications. Based on these results, ResNet34 was chosen for the DLG model because the observed classification performance was optimal among the several deep-learning models. A more intuitive comparison is provided by the ROC curves of the multitasking classification shown in Figure 4.

3.3. Interpretability of the DLG Model. Setting a threshold of $p<0.05$, more than ten thousand SNP loci showed differences between the groups, and even the significance of the most frequently identified loci was below 0.001 .

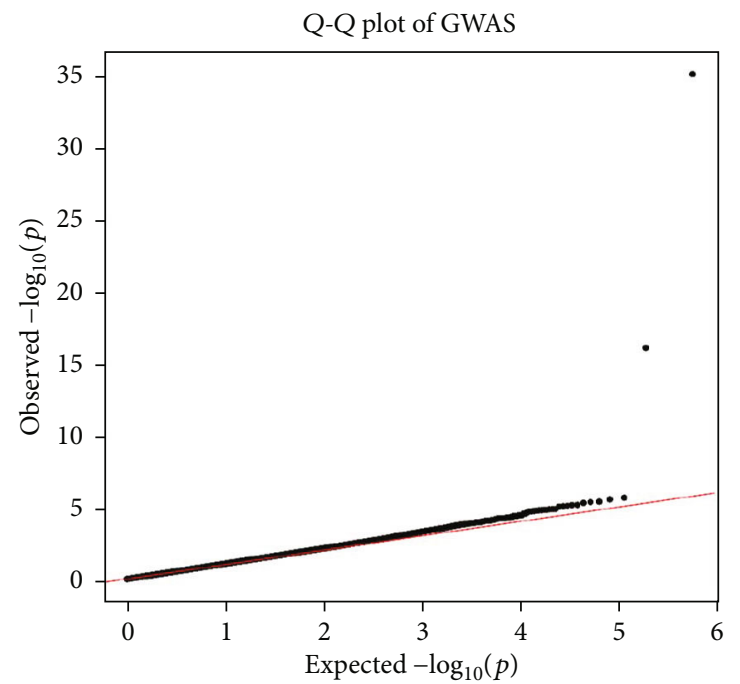

FIgURE 3: $Q-Q$ plot of genome-wide association study (GWAS) between $\mathrm{AD}$ and $\mathrm{HC}$ groups. Genomic inflation factor is 1.084 .

Firstly, we compared the significant SNPs with those previously identified by GWAS as genetic susceptibility factors. Almost one hundred SNP loci between AD patients and HCs were consistent with findings from previous studies. Likewise, more than one hundred associated SNP loci were also found between the $\mathrm{AD}$ and MCI groups and between the $\mathrm{HC}$ and MCI groups.

Secondly, we sought significant SNP loci among three classification tasks. The gene regions of sixty-six SNP loci were shared in different stages of $\mathrm{AD}$ progression. Table 4 summarizes the sixty-six shared significant SNP loci among the three classifications, including, e.g., the well-known CLU, PICALM, and SORL1 gene regions. For rs11136000 (CLU) in chromosome 8 , its $p$ values were $6.63 e^{-4}$ between the $\mathrm{AD}$ and $\mathrm{HC}$ groups, $8.37 e^{-6}$ between the MCI and $\mathrm{HC}$ groups, and $1.49 e^{-7}$ between MCI and AD groups. In addition, three SNP loci, rs543293, rs10501602, and rs3851179, were found in the PICALM gene region of chromosome 11, and the $p$ values of rs3851179 for the comparisons of the three groups were $6.00 e^{-3}, 1.06 e^{-6}$, and $1.51 e^{-20}$, while the $p$ 
TABLE 2: SNP summaries reaching genome-wide significance after GWAS.

\begin{tabular}{lcccccc}
\hline SNP & Position & Chr & Region or closest gene & Major/minor alleles & $p$ value & OR \\
\hline rs429358 & 44908684 & 19 & APOE & C/T & $5.407 e-36$ & 4.348 \\
rs2075650 & 50087459 & 19 & TOMM40 & G/A & $7.19 e-17$ & 2.737 \\
\hline
\end{tabular}

$p<1.66 e^{-7}$ for SNPs listed above. Chr: chromosome.

TABle 3: Performance of different classification approaches in multitasking classification.

\begin{tabular}{lccc}
\hline Model & Accuracy (\%) & Sensitivity (\%) & Specificity (\%) \\
\hline AD and HC groups & & & AUC \\
GWAS analysis & $71.38 \pm 0.63$ & $63.13 \pm 2.87$ & $85.59 \pm 6.66$ \\
CNN model & $92.45 \pm 8.13$ & $93.87 \pm 12.26$ & $90.00 \pm 15.97$ \\
ResNet18 & $97.96 \pm 1.71$ & $97.42 \pm 3.16$ & $98.89 \pm 1.36$ \\
ResNet34 & $98.78 \pm 1.50$ & $98.39 \pm 2.50$ & $99.44 \pm 1.11$ \\
MCI and HC groups & & & 0.915 \\
GWAS analysis & $56.99 \pm 1.55$ & $96.08 \pm 13.92$ & $5.94 \pm 21.65$ \\
CNN model & $87.47 \pm 16.64$ & $99.57 \pm 0.85$ & $71.67 \pm 38.75$ \\
ResNet18 & $97.59 \pm 3.73$ & $100.00 \pm 0.00$ & $94.44 \pm 8.61$ \\
ResNet34 & $99.52 \pm 0.60$ & $99.57 \pm 0.85$ & $99.44 \pm 1.11$ \\
AD and MCI groups & & & 0.510 \\
GWAS analysis & $58.97 \pm 0.00$ & $72.18 \pm 0.01$ & 0.852 \\
CNN model & $86.42 \pm 16.02$ & $97.42 \pm 4.40$ & 0.966 \\
ResNet18 & $97.80 \pm 1.24$ & $97.74 \pm 2.41$ & 0.986 \\
ResNet34 & $98.90 \pm 1.78$ & $100.00 \pm 0.00$ & $71.54 \pm 0.01$ \\
\hline
\end{tabular}

The methods are conducted with crossvalidation, and their results are given as mean \pm standard deviation.

values of rs543293 were $4.65 e^{-3}, 3.43 e^{-8}$, and $2.27 e^{-13}$, and the $p$ values of rs3851179 were also much less than 0.001 . These results are well supported by previous studies. Other significant results are detailed in Table 4, and the heatmaps of significant SNPs in chromosomes 8, 11, and 13 are shown in Figure 5. The horizontal axis represents major and minor alleles, and the vertical axis represents the $p$ value of SNP loci in the chromosomes. We observed some distinct differences, for example, between rs11136000, rs3851179, and surrounding loci.

In addition, except for those in Table 4, there were also several SNP loci showing an association with AD progression in their respective classifications. Several also have been reported and confirmed in previous large-scale GWAS studies, including APOE, BIN1, CHRM1, and TOMM40 with $p$ values much less than 0.001 . Furthermore, it is notable that rs6311 and rs6313 in the HTR2A gene region, rs1354269 in the NAV2 gene, and rs690705 in the RFC3 gene all exhibited significant differences among the three classifications. For instance, the $p$ values of rs6311 were $1.96 e^{-5}, 2.52 e^{-3}$, and $1.48 e^{-11}$ between the respective groups, and the $p$ values of rs6313 were $3.21 e^{-5}, 4.55 e^{-3}$, and $2.05 e^{-12}$. An understanding of the roles of these novel loci in $\mathrm{AD}$ requires future study.

All of the information above was deposited in the DisGeNET database (http://www.disgenet.org/home/), a discovery platform containing one of the largest publicly available collections of genes and variants associated with human disease.

\section{Discussion}

This study used a comparison of the performance of several different deep-learning models as a basis for proposing a deep-learning genomics method based on ResNet34. The classification results indicate that the DLG model offers a higher diagnostic value than traditional GWAS analysis.

4.1. Outcomes of GWAS Analysis. In GWAS analyses, two SNPs have been identified at the $p<1.66 e^{-7}$ significance level: APOE SNP rs429358 was determined to be the most significant genetic risk factor for AD. And the second most significant factor, TOMM40 SNP rs2075650, was found to be adjacent to the APOE SNP [10]. These results are consistent with previous studies. Although these SNP loci were identified by GWAS, traditional GWAS analysis suffers from being influenced by small sample size. Because other common genetic risk factors may have a much smaller impact on risk than the APOE gene, novel risk factors present in small samples may go undetected by GWAS analysis. Several previous studies have also demonstrated an explicit relationship between sample size and the number of significant 


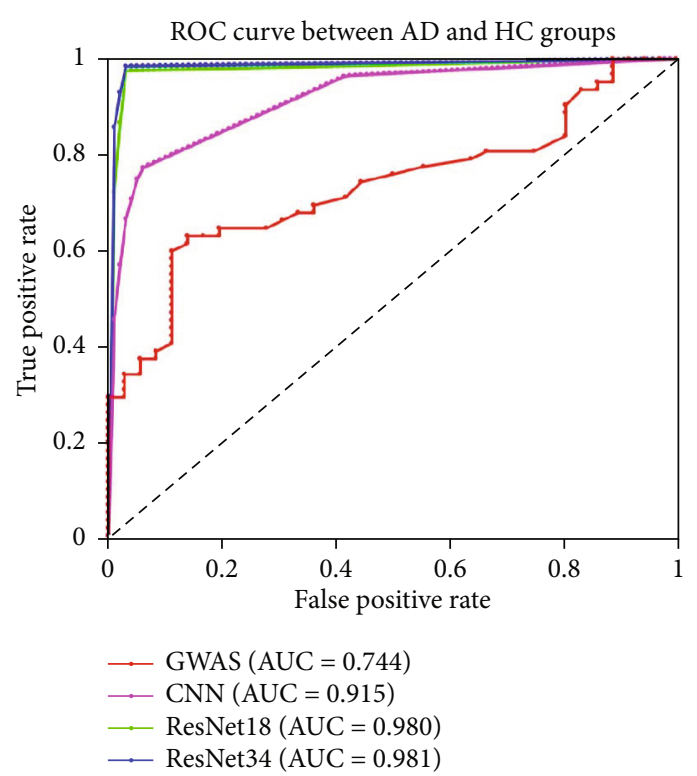

(a)

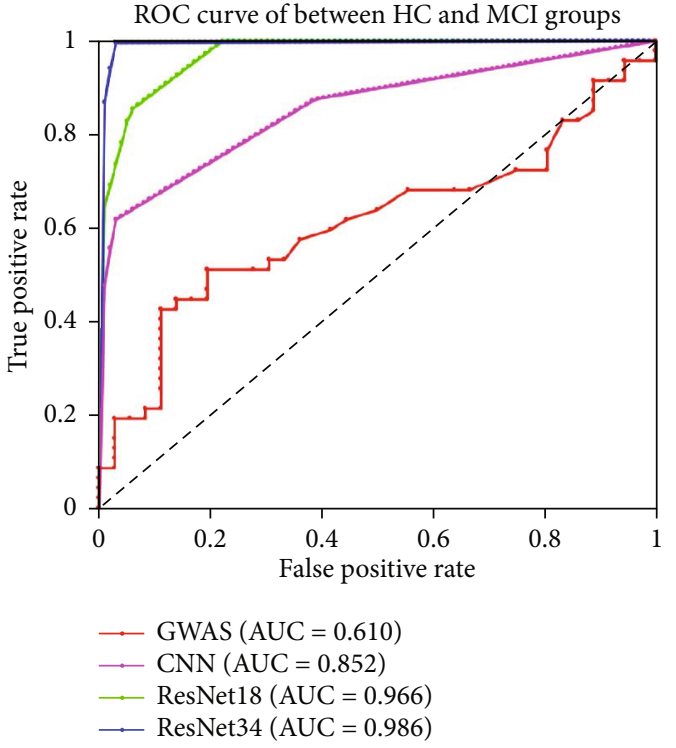

(b)

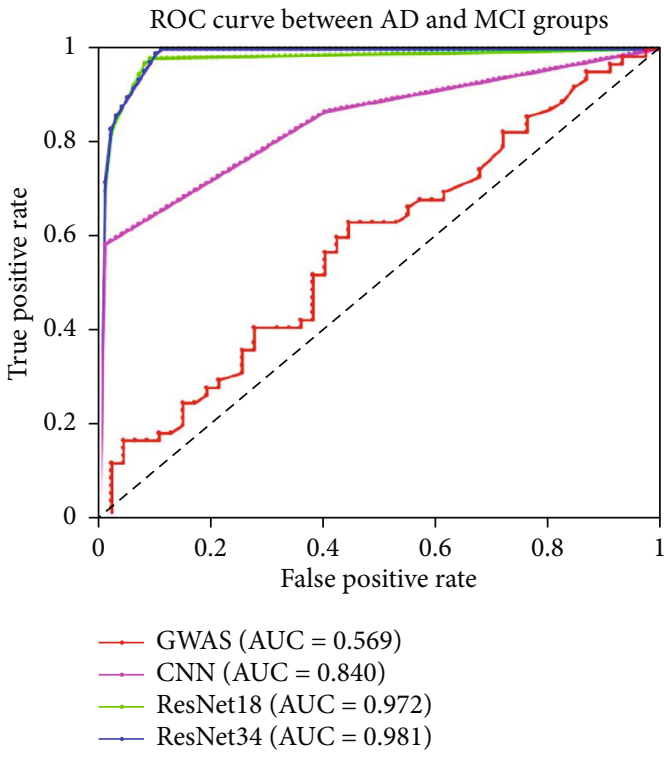

(c)

FIGURE 4: ROC curve of the performance of different classification approaches in multitasking classification. (a) ROC curve between AD and HC groups. (b) ROC curve between MCI and HC groups. (c) ROC curve between AD and MCI groups.

differences in traits identified by genome-wide association studies $[18,19]$.

4.2. Classification Performance. In this study, in order to construct a deep-learning genomics model, we compared the performance of several deep-learning classification methods, including a simple CNN model, ResNet18, and ResNet34. As shown in Table 3, we observed that the results of the deep residual network were superior to those of a simple CNN, and in the process of training the model, the ResNet models exhibited robustness and stability superior to those of CNNs, and furthermore, ResNet34 was superior to RseNet18. Therefore, we chose ResNet34 as the final DLG model. More importantly, we compared the performance of the DLG model and traditional GWAS analysis under the same conditions and found the classification results of the DLG model to be superior. These results suggest that the deep-learning algorithm is effective in genome applications and that development of deep-learning genomics is worthy of further exploration.

4.3. Interpretability of the DLG Model. When we interpreted the DLG model, we found more than one thousand SNP loci with significant differences between $\mathrm{AD}$ patients and $\mathrm{HCs}$, between the MCI and AD groups, and between the $\mathrm{HC}$ and MCI subjects. As is well known, rs11136000 (CLU), rs3851179 (PICALM), rs2070045 (SORL1), and rs1699102 (SORL1) have previously been identified as risk factors for 
TABLE 4: Shared significant SNPs in AD multitasking classification at $p$ threshold of 0.05 .

\begin{tabular}{|c|c|c|c|c|c|c|c|}
\hline SNP loci & Chr & Position & Region or closest gene & Major/minor alleles & $\begin{array}{c}p \text { value } \\
\text { HC vs. AD }\end{array}$ & $\begin{array}{c}p \text { value } \\
\mathrm{HC} \text { vs. } \mathrm{MCI}\end{array}$ & $\begin{array}{c}p \text { value } \\
\text { AD vs. } \mathrm{MCI}\end{array}$ \\
\hline rs12091371 & 1 & 238671675 & FMN2 & $\mathrm{A} / \mathrm{G}$ & 0.002227833 & 0.001509792 & $2.27881 E-09$ \\
\hline rs12129547 & 1 & 238761878 & GREM2 & $\mathrm{T} / \mathrm{C}$ & 0.002767757 & 0.019531535 & $3.93396 E-11$ \\
\hline rs1801131 & 1 & 11777063 & MTHFR & $\mathrm{C} / \mathrm{A}$ & 0.000154746 & 0.007333869 & $6.18756 E-21$ \\
\hline rs1801133 & 1 & 11778965 & MTHFR & $\mathrm{T} / \mathrm{C}$ & 0.000192989 & 0.009566143 & $5.35043 E-21$ \\
\hline rs 17034806 & 2 & 109002337 & RANBP2 & G/A & 0.002645921 & 0.028425309 & $3.04207 E-10$ \\
\hline rs243034 & 2 & 60456396 & MIR4432HG & G/A & 0.038985718 & 0.009492742 & $1.40355 E-08$ \\
\hline rs4676049 & 2 & 109001689 & RANBP2 & $\mathrm{T} / \mathrm{C}$ & 0.002215778 & 0.034993277 & $4.51555 E-10$ \\
\hline rs6714710 & 2 & 97711518 & ZAP70 & $\mathrm{G} / \mathrm{T}$ & 0.038909198 & 0.000768783 & $7.036 E-09$ \\
\hline rs 1498853 & 3 & 69691797 & $\mathrm{NAN}^{\mathrm{a}}$ & $\mathrm{G} / \mathrm{A}$ & 0.000708166 & 0.012435495 & $6.43721 E-09$ \\
\hline rs2289506 & 3 & 101547592 & NIT2 & $\mathrm{T} / \mathrm{C}$ & 0.000412705 & 0.004914541 & $1.07429 E-18$ \\
\hline rs288496 & 3 & 69714739 & $\mathrm{NAN}^{\mathrm{a}}$ & $\mathrm{T} / \mathrm{C}$ & 0.002172998 & 0.009409715 & $4.5892 E-09$ \\
\hline rs3864101 & 3 & 188862449 & $\mathrm{NAN}^{\mathrm{a}}$ & $\mathrm{T} / \mathrm{G}$ & 0.038241862 & 0.022506051 & $6.17468 E-08$ \\
\hline rs989692 & 3 & 156284059 & MME & $\mathrm{T} / \mathrm{C}$ & 0.00243336 & 0.016131413 & $5.91293 E-09$ \\
\hline rs3796529 & 4 & 57492171 & REST & $\mathrm{A} / \mathrm{G}$ & 0.048696066 & 0.004826601 & $7.08294 E-25$ \\
\hline rs753129 & 4 & 56363188 & $\mathrm{NAN}^{\mathrm{a}}$ & $\mathrm{C} / \mathrm{T}$ & 0.004469287 & $1.23015 E-10$ & 0.013855564 \\
\hline rs1925458 & 6 & 23486930 & LOC102724749; LOC105374976 & $\mathrm{T} / \mathrm{G}$ & 0.000567167 & 0.000409483 & $3.10404 E-11$ \\
\hline rs1980493 & 6 & 32471193 & BTNL2; TSBP1-AS1 & G/A & 0.016858811 & 0.000751139 & $3.35304 E-14$ \\
\hline rs2651206 & 6 & 43321455 & TTBK1 & $\mathrm{T} / \mathrm{C}$ & 0.034368574 & $2.23746 E-07$ & $1.97506 E-11$ \\
\hline rs3734254 & 6 & 35502988 & PPARD & $\mathrm{C} / \mathrm{T}$ & 0.001667544 & 0.000173601 & $2.21574 E-11$ \\
\hline rs3747742 & 6 & 41270496 & TREML2 & $\mathrm{C} / \mathrm{T}$ & $6.7184 E-06$ & $3.50103 E-05$ & $1.19671 E-08$ \\
\hline rs6455128 & 6 & 62755705 & KHDRBS2 & $\mathrm{A} / \mathrm{C}$ & 0.008767325 & 0.00013524 & $1.45915 E-14$ \\
\hline rs11767557 & 7 & 142819261 & EPHA1-AS1 & $\mathrm{C} / \mathrm{T}$ & 0.001139233 & $1.20661 E-07$ & $1.96401 E-07$ \\
\hline rs11771145 & 7 & 142820884 & EPHA1-AS1 & $\mathrm{A} / \mathrm{G}$ & 0.001112928 & $9.62693 E-08$ & $1.8557 E-07$ \\
\hline rs2227631 & 7 & 100556258 & SERPINE1 & G/A & 0.003024669 & $8.25065 E-08$ & $7.24385 E-07$ \\
\hline rs6461569 & 7 & 21502301 & SP4 & $\mathrm{C} / \mathrm{T}$ & 0.025204688 & $1.41338 E-06$ & 0.001322394 \\
\hline rs6966915 & 7 & 12232513 & TMEM106B & $\mathrm{T} / \mathrm{C}$ & 0.031810788 & 0.004328052 & $6.57464 E-09$ \\
\hline rs11136000 & 8 & 27520436 & CLU & $\mathrm{T} / \mathrm{C}$ & 0.000663408 & $8.36729 E-06$ & $1.48921 E-07$ \\
\hline rs1975804 & 8 & 109360409 & EIF3E; LOC105375704 & $\mathrm{C} / \mathrm{T}$ & 0.000833268 & 0.003779274 & 0.040135088 \\
\hline rs1800977 & 9 & 106730271 & ABCA1; LOC105376196 & $\mathrm{T} / \mathrm{C}$ & 0.013227434 & $5.61278 E-05$ & $4.22923 E-14$ \\
\hline rs2007153 & 9 & 135493640 & $\mathrm{DBH}$ & $\mathrm{A} / \mathrm{G}$ & $3.88584 E-05$ & 0.000993637 & $4.15766 E-07$ \\
\hline rs2066715 & 9 & 106627854 & ABCA1 & $\mathrm{A} / \mathrm{G}$ & 0.004157538 & 0.04271672 & $7.79217 E-05$ \\
\hline rs2283123 & 9 & 135505118 & $\mathrm{DBH}$ & $\mathrm{T} / \mathrm{C}$ & 0.000105332 & 0.00221966 & $1.99182 E-07$ \\
\hline rs2740483 & 9 & 106730356 & ABCA1; LOC105376196 & $\mathrm{C} / \mathrm{G}$ & 0.009678488 & $6.53287 E-05$ & $3.81638 E-14$ \\
\hline rs4149313 & 9 & 106626574 & ABCA1 & $\mathrm{G} / \mathrm{A}$ & 0.003318514 & 0.040059489 & $7.22211 E-05$ \\
\hline rs4878104 & 9 & 89382811 & DAPK1 & $\mathrm{T} / \mathrm{C}$ & 0.002309724 & $6.11706 E-05$ & $1.46237 E-14$ \\
\hline rs4548513 & 10 & 67710331 & CTNNA3 & $\mathrm{T} / \mathrm{C}$ & 0.004692221 & 0.000992064 & $6.62732 E-08$ \\
\hline rs7070570 & 10 & 67534610 & LOC105378340; CTNNA3 & $\mathrm{G} / \mathrm{A}$ & 0.000655491 & $2.34147 E-05$ & $1.63609 E-06$ \\
\hline rs10501602 & 11 & 85359037 & PICALM & $\mathrm{G} / \mathrm{A}$ & 0.004647595 & $3.42511 E-08$ & $2.27238 E-13$ \\
\hline rs1133174 & 11 & 121006965 & SORL1 & $\mathrm{A} / \mathrm{G}$ & 0.010544659 & 0.003255318 & $3.13279 E-05$ \\
\hline rs 12805520 & 11 & 85308059 & CCDC83 & $\mathrm{T} / \mathrm{C}$ & 0.007162755 & $4.02484 E-08$ & $1.90295 E-11$ \\
\hline rs1354269 & 11 & 19330820 & NAV2 & $\mathrm{C} / \mathrm{T}$ & 0.010833795 & $6.42781 E-08$ & $2.65216 E-24$ \\
\hline rs 1695 & 11 & 67109265 & GSTP1 & G/A & 0.006309008 & $6.1645 E-07$ & $2.06528 E-23$ \\
\hline rs1699102 & 11 & 120962172 & SORL1 & $\mathrm{C} / \mathrm{T}$ & 0.013141699 & 0.001477832 & $6.19383 E-05$ \\
\hline rs17571 & 11 & 1739170 & CTSD & $\mathrm{T} / \mathrm{C}$ & 0.013910348 & $1.48442 E-08$ & $1.58542 E-20$ \\
\hline rs1946518 & 11 & 111540668 & IL18 & $\mathrm{T} / \mathrm{G}$ & 0.006486007 & $1.8075 E-06$ & $7.21291 E-22$ \\
\hline rs2070045 & 11 & 120953300 & SORL1 & $\mathrm{G} / \mathrm{T}$ & 0.014545915 & 0.001140944 & $7.97485 E-05$ \\
\hline rs3851179 & 11 & 85546288 & PICALM & $\mathrm{A} / \mathrm{G}$ & 0.00599555 & $1.06305 E-06$ & $1.50773 E-20$ \\
\hline rs543293 & 11 & 85497725 & PICALM & $\mathrm{A} / \mathrm{G}$ & 0.004782682 & $1.26328 E-07$ & $7.85454 E-18$ \\
\hline
\end{tabular}


TABLE 4: Continued.

\begin{tabular}{|c|c|c|c|c|c|c|c|}
\hline SNP loci & Chr & Position & Region or closest gene & Major/minor alleles & $\begin{array}{c}p \text { value } \\
\mathrm{HC} \text { vs. AD }\end{array}$ & $\begin{array}{c}p \text { value } \\
\mathrm{HC} \text { vs. } \mathrm{MCI}\end{array}$ & $\begin{array}{c}p \text { value } \\
\text { AD vs. } \mathrm{MCI}\end{array}$ \\
\hline rs6265 & 11 & 27636492 & BDNF; BDNF-AS & $\mathrm{A} / \mathrm{G}$ & 0.009460455 & $1.96067 E-07$ & $1.42052 E-28$ \\
\hline rs7120118 & 11 & 47242866 & NR1H3 & $\mathrm{C} / \mathrm{T}$ & 0.034010947 & 0.011720707 & $5.90652 E-22$ \\
\hline rs7943454 & 11 & 24478242 & LUZP2 & $\mathrm{T} / \mathrm{C}$ & 0.01777448 & $1.41141 E-06$ & $2.00064 E-30$ \\
\hline rs1799986 & 12 & 55821533 & LRP1 & $\mathrm{T} / \mathrm{C}$ & 0.000875404 & $8.1991 E-06$ & 0.000948497 \\
\hline rs6311 & 13 & 46369479 & HTR2A & $\mathrm{T} / \mathrm{C}$ & $1.95644 E-05$ & 0.002519461 & $1.48261 E-11$ \\
\hline rs6313 & 13 & 46367941 & HTR2A & $\mathrm{T} / \mathrm{C}$ & $3.21121 E-05$ & 0.004551786 & $2.05426 E-12$ \\
\hline rs690705 & 13 & 33552918 & RFC3 & $\mathrm{G} / \mathrm{A}$ & $9.15016 E-07$ & 0.000123939 & 0.032894005 \\
\hline rs7989332 & 13 & 19948575 & CRYL1 & $\mathrm{A} / \mathrm{C}$ & 0.001947338 & 0.038324875 & 0.017831896 \\
\hline rs10137185 & 14 & 63845529 & ESR2 & $\mathrm{T} / \mathrm{C}$ & 0.000105484 & 0.00099597 & $2.08051 E-22$ \\
\hline rs 1065778 & 15 & 49307498 & MIR4713HG; CYP19A1 & $\mathrm{A} / \mathrm{G}$ & $7.88465 E-05$ & $1.51664 E-05$ & $2.28151 E-18$ \\
\hline rs2278317 & 15 & 31848032 & RYR3 & $\mathrm{G} / \mathrm{A}$ & 0.019133823 & $5.18518 E-05$ & $4.11594 E-07$ \\
\hline rs3751592 & 15 & 49393870 & CYP19A1; MIR7973-1; MIR7973-2 & $\mathrm{G} / \mathrm{A}$ & 0.000333452 & $8.25536 E-05$ & $5.77922 E-13$ \\
\hline rs11075996 & 16 & 52415525 & FTO & $\mathrm{T} / \mathrm{C}$ & 0.021538201 & $9.92354 E-07$ & 0.002856803 \\
\hline rs11075997 & 16 & 52416413 & FTO & $\mathrm{T} / \mathrm{C}$ & 0.023256237 & $1.14854 E-06$ & 0.002461173 \\
\hline rs6499640 & 16 & 52327178 & FTO & $\mathrm{G} / \mathrm{A}$ & 0.016928027 & $4.22569 E-07$ & 0.009148135 \\
\hline rs 1050565 & 17 & 25600202 & BLMH & $\mathrm{G} / \mathrm{A}$ & 0.045066195 & $6.1136 E-06$ & $1.19122 E-06$ \\
\hline rs391300 & 17 & 2163008 & SRR & $\mathrm{A} / \mathrm{G}$ & 0.028223591 & $1.90848 E-06$ & 0.000379785 \\
\hline rs7946 & 17 & 17350285 & PEMT & $\mathrm{C} / \mathrm{T}$ & 0.044709718 & 0.000412862 & $2.75631 E-18$ \\
\hline
\end{tabular}

SNP: single-nucleotide polymorphism. Chr: chromosome. NAN ${ }^{a}$ represents uncertain gene of the SNP loci. $p$ value (HC vs. AD) represents the difference value of each SNP loci between AD and HC groups. All $p$ value are calculated by a two-sample $t$ test for false discovery rate correction.

$\mathrm{AD}[7,9,39]$. Notably, they were all included among the sixty-six significant SNP loci shared in the three classification tasks in this study (as shown in Table 4 and Figure 5). For example, previous studies have shown that CLU modulates $\mathrm{A} \beta$ metabolism and is involved in $\mathrm{A} \beta$ clearance or acts as a chaperon for protein degradation [40]. PICALM, as an adaptor protein involved in clathrin-mediated endocytosis, regulates amyloid precursor protein (APP) internalization and subsequent $\mathrm{A} \beta$ generation, contributing to brain amyloid plaque load via its effect on $\mathrm{A} \beta$ metabolism $[41,42]$. In addition to the analysis of the above identical SNP loci found among the three classification tasks, several differential loci were identified among one or two classification tasks, which are also consistent with previous research. Rs10194375 (BIN1), a protein that may be associated with tau-mediated pathology was identified as being significant between the $\mathrm{AD}$ and $\mathrm{HC}$ groups and the $\mathrm{AD}$ and $\mathrm{MCI}$ groups. In addition, rs2075650 (TOMM40), rs405509 (APOE), and rs429358 (APOE) were identified as significant between the $\mathrm{HC}$ and $\mathrm{MCI}$ groups and the MCI and AD groups. In summary, the DLG model is able to identify differential genomics in multitasking classification.

Most importantly, in addition to those shown to associate with $\mathrm{AD}$ in the past, we found several new SNP loci, including rs6311 (HTR2A), rs6313 (HTR2A), rs1354269 (NAV2), rs1946518 (IL18), rs1799986 (LRP1), rs690705 (RFC3), and rs7943454 (LUZP2), whose $p$ values were highly significan$\mathrm{t}$ (as shown in Table 4). Rs6311 and rs6313 are in the HTR2A gene region. The HTR2A gene in humans is located on chromosome 13 and consists of exons separated by only two introns and encodes one of the receptors for serotonin. According to previous publications, HTR2A has received much attention in many psychiatric disorders such as mood disorders, attention deficit hyperactivity disorder, anxiety disorders, and schizophrenia. On the one hand, some studies have shown that medications for mood disorders and related conditions work by blocking 5-HT2A and altering the function of certain brain circuits. And blocking 5-HTR2A also seems to improve the effects of some antidepressants [43]. On the other hand, the numbers of the postsynaptic receptor HTR2A are reduced in the neocortex, and it seems to be involved in memory via its role in cortical pyramidal cells. For example, in AD research, HTR2A receptor densities in the brains of $\mathrm{AD}$ subjects were found to be reduced compared with age-matched controls, and the researchers also found this reduction correlated with the rate of decline of cognitive scores [44]. Hence, since subjects with AD or mild cognitive impairment exhibit depression and anxiety to various degrees, it is worth exploring whether rs6311 and rs6313 of the HTR2A gene contribute to AD susceptibility. Another significant locus identified here was rs1354269 located in the NAV2 gene region. The NAV2 gene, which encodes a member of the neuron navigator gene family, is highly expressed in brain and is involved in the development of the nervous system. Hence, the role of the NAV2 gene in $\mathrm{AD}$ is also worthy of future investigation. In addition, rs 690705 of the RFC3 gene region also exhibited a significant difference in group-level classifications, and its impact on $\mathrm{AD}$ should be examined in the future.

\section{Limitations}

It is worth noting some limitations of this study. Firstly, only gene sequences were used as inputs to the DLG classification. 


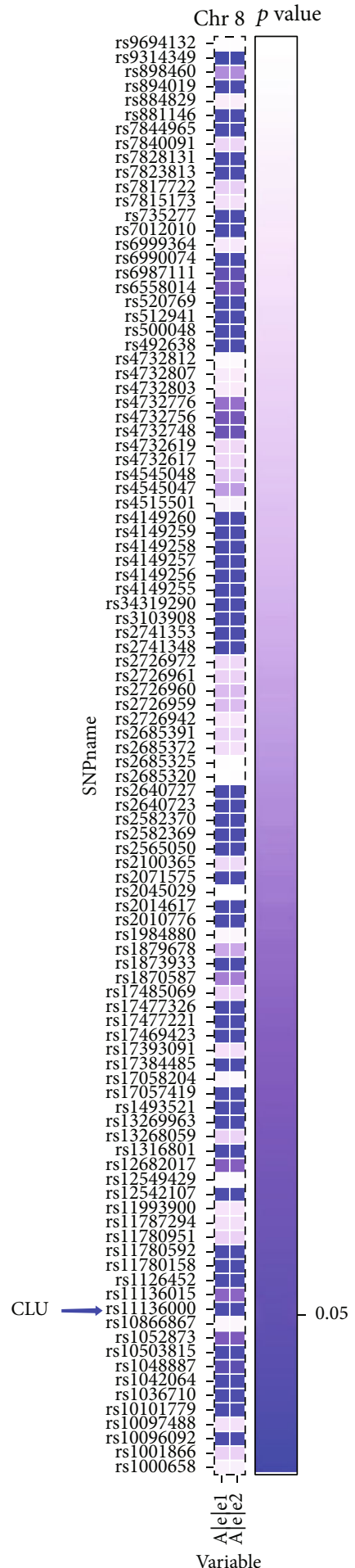

(a)

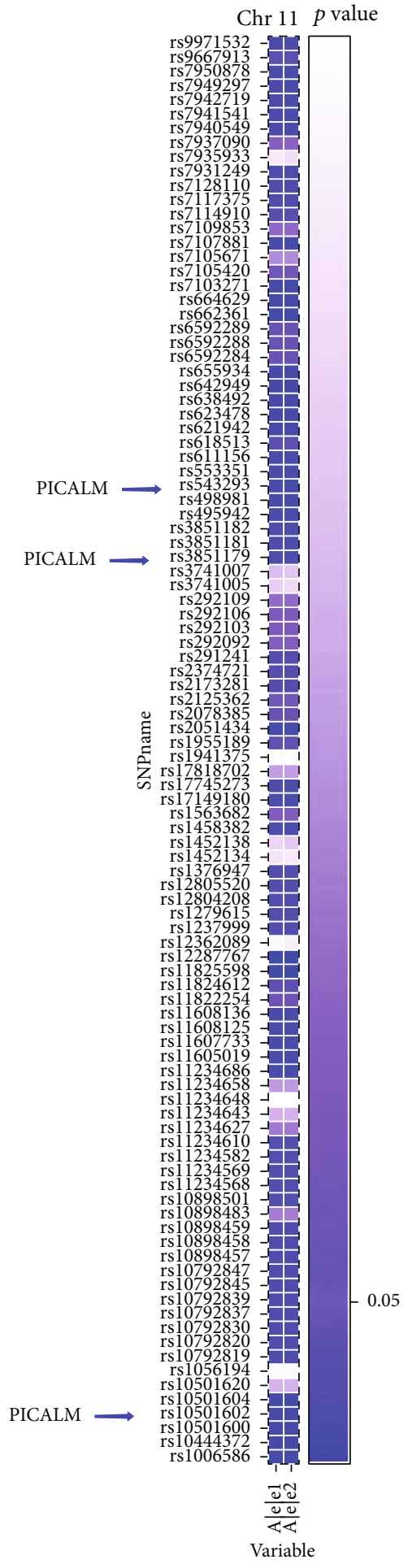

(b)

Figure 5: Continued. 


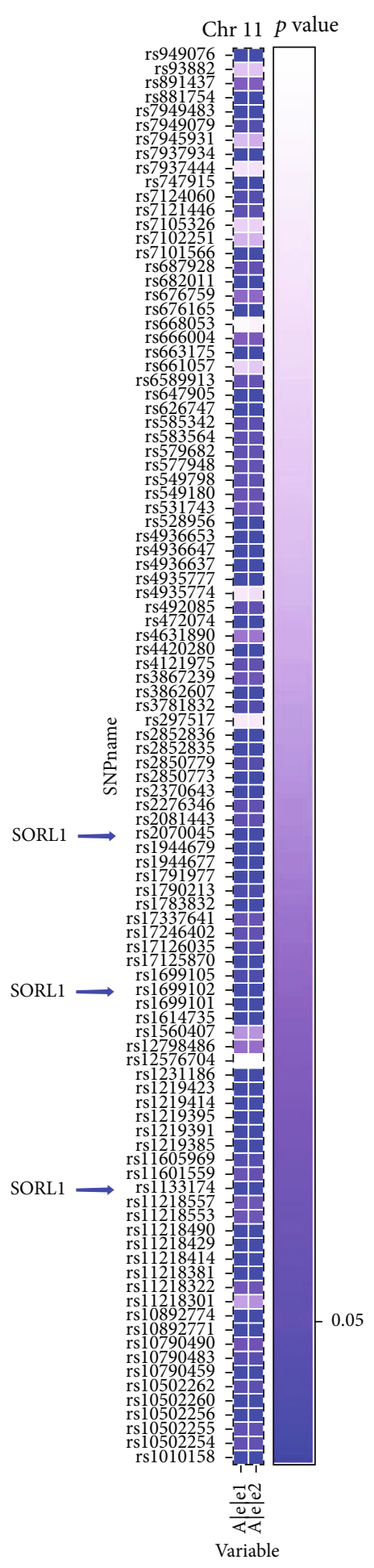

(c)

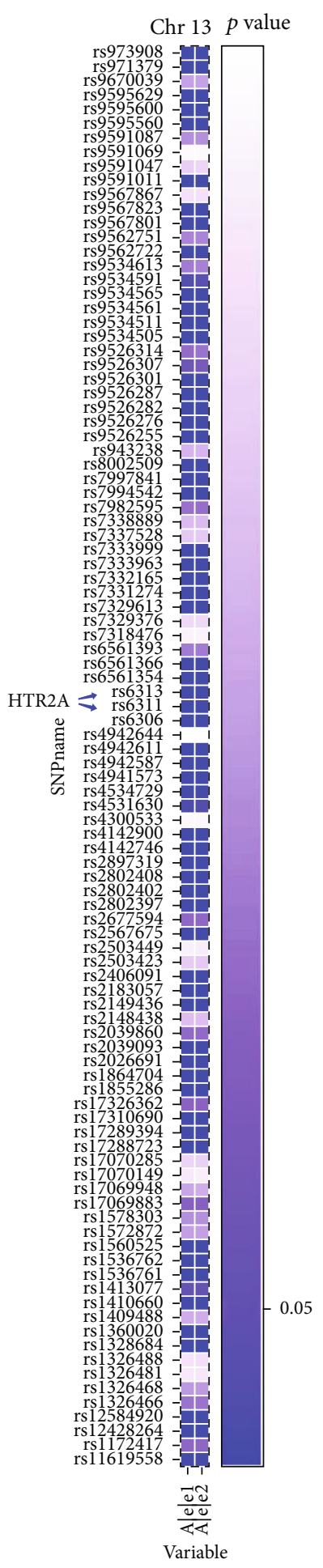

(d)

FIgURE 5: Visualization for part of shared significant SNPs in AD multitasking classification at $p$ threshold of 0.05 . Rs11136000 (CLU) on chromosome 8 (a); rs543293, rs10501602, and rs3851179 (PICALM) on chromosome 11 (b); rs2070045, rs1699102, and rs1133174 (SORL1) on chromosome 11 (c); and rs6311 and rs6313 (HTR2A) on chromosome 13 (d) are shown successively from left to right. 
In the future work, we plan to combine gene sequences with clinical data and brain imaging [45] to facilitate investigation of the mechanisms of $\mathrm{AD}$ progression by deep-learning genomics and deep-learning radiomics approaches. Secondly, we only classified information from the ADNI dataset in this study, so the results could be strengthened by including other datasets such as the Chinese populations. Thirdly, the number of subjects represented in this study may be limiting. Lastly, although this study has demonstrated the feasibility of DLG approach, it will be important to further explore the interpretability of deep-learning genomics.

\section{Conclusions}

In conclusion, the current study suggests that the deeplearning genomics approach is effective for multitasking classification research on $\mathrm{AD}$ progression and outperforms traditional GWAS analysis. Moreover, the several novel SNP loci identified in the DLG approach including rs6311 and rs6313 in HTR2A, rs1354269 in NAV2, and rs690705 in RFC3 are worthy of further exploration to better understand the mechanisms of $\mathrm{AD}$.

\section{Data Availability}

The datasets presented in this study were obtained from ADNI (http://adni.loni.usc.edu/).

\section{Conflicts of Interest}

The authors declare that there is no conflict of interest regarding the publication of this paper.

\section{Authors' Contributions}

Lanlan Li and Yeying Yang contributed equally to this work.

\section{Acknowledgments}

Data collection and dissemination for this project were funded by the Alzheimer's Disease Neuroimaging Initiative (ADNI): the National Institutes of Health (grant number U01 AG024904) and the Department of Defense (award number W81XWH-12-2-0012). ADNI is funded by the National Institute of Aging and the National Institute of Biomedical Imaging and Bioengineering as well as through generous contributions from the following organizations: AbbVie, Alzheimer's Association, Alzheimer's Drug Discovery Foundation, Araclon Biotech, BioClinica Inc., Biogen, Bristol-Myers Squibb Company, CereSpir Inc., Eisai Inc., Elan Pharmaceuticals Inc., Eli Lilly and Company, EuroImmun, F. Hoffmann-La Roche Ltd. and its affiliated company Genentech Inc., Fujirebio, GE Healthcare, IXICO Ltd., Janssen Alzheimer Immunotherapy Research \& Development LLC., Johnson \& Johnson Pharmaceutical Research \& Development LLC., Lumosity, Lundbeck, Merck \& Co. Inc., Meso Scale Diagnostics LLC., NeuroRx Research, Neurotrack Technologies, Novartis Pharmaceuticals Corporation, Pfizer Inc., Piramal Imaging, Servier, Takeda Pharmaceutical Company, and Transition Therapeutics. The Canadian Institutes of Health Research are providing funds to support ADNI clinical sites in Canada. Private sector contributions are facilitated by the Foundation for the National Institutes of Health (http://www.fnih.org/). The grantee organization is the Northern California Institute for Research and Education, and the study is coordinated by the Alzheimer's Disease Cooperative Study at the University of California, San Diego, CA, USA. ADNI data are disseminated by the Laboratory for Neuro Imaging at the University of Southern California, CA, USA. This study was supported by grants received from the National Natural Science Foundation of China (grant numbers 82020108013); the 111 Project (grant number D20031); the Shanghai Municipal Science and Technology Major Project (grant number 2017SHZDZX01).

\section{References}

[1] “2019 Alzheimer's disease facts and figures," Alzheimer's \& Dementia, vol. 15, no. 3, pp. 321-387, 2019.

[2] “2020 Alzheimer's disease facts and figures," Alzheimer's \& Dementia, vol. 16, no. 3, pp. 391-460, 2020.

[3] B. Dubois, H. Hampel, H. H. Feldman et al., "Preclinical Alzheimer's disease: definition, natural history, and diagnostic criteria," Alzheimer's \& Dementia, vol. 12, no. 3, pp. 292-323, 2016.

[4] Q. Y. Dong, T. R. Li, X. Y. Jiang, X. N. Wang, Y. Han, and J. H. Jiang, "Glucose metabolism in the right middle temporal gyrus could be a potential biomarker for subjective cognitive decline: a study of a Han population," Alzheimer's Research \& Therapy, vol. 13, no. 1, p. 74, 2021.

[5] Y. Huang and L. Mucke, "Alzheimer mechanisms and therapeutic strategies," Cell, vol. 148, no. 6, pp. 1204-1222, 2012.

[6] C. M. Karch, C. Cruchaga, and A. M. Goate, "Alzheimer's disease genetics: from the bench to the clinic," Neuron, vol. 83, no. 1, pp. 11-26, 2014.

[7] D. Harold, R. Abraham, P. Hollingworth et al., "Erratum: Genome-wide association study identifies variants at CLU and PICALM associated with Alzheimer's disease," Nature Genetics, vol. 41, no. 10, p. 1156, 2009.

[8] A. Montagne, D. A. Nation, A. P. Sagare et al., "APOE4 leads to blood -brain barrier dysfunction predicting cognitive decline," Nature, vol. 581, no. 7806, pp. 71-76, 2020.

[9] B. N. Vardarajan, Y. Zhang, J. H. Lee et al., "Coding mutations inSORL1and Alzheimer disease," Annals of Neurology, vol. 77, no. 2, pp. 215-227, 2015.

[10] H. Huang, J. Zhao, B. Xu et al., "The TOMM40 gene rs2075650 polymorphism contributes to Alzheimer's disease in Caucasian, and Asian populations," Neuroscience Letters, vol. 628, pp. 142-146, 2016.

[11] N. R. Wray, M. E. Goddard, and P. M. Visscher, "Prediction of individual genetic risk of complex disease," Current Opinion in Genetics \& Development, vol. 18, no. 3, pp. 257-263, 2008.

[12] V. Escott-Price, C. Bellenguez, L. S. Wang et al., "Gene-wide analysis detects two new susceptibility genes for Alzheimer's disease," PLoS One, vol. 9, no. 6, article e94661, 2014.

[13] V. Escott-Price, R. Sims, C. Bannister et al., "Common polygenic variation enhances risk prediction for Alzheimer's disease," Brain, vol. 138, no. 12, pp. 3673-3684, 2015.

[14] the Alzheimer's Disease Neuroimaging Initiative, CHARGE consortium, EADI1 consortium et al., "Common variants at 
ABCA7, MS4A6A/MS4A4E, EPHA1, CD33 and CD2AP are associated with Alzheimer's disease," Nature Genetics, vol. 43, no. 5, pp. 429-435, 2011.

[15] Alzheimer's Disease Sequencing Project, J. C. Bis, X. Jian et al., "Whole exome sequencing study identifies novel rare and common Alzheimer's-associated variants involved in immune response and transcriptional regulation," Molecular Psychiatry, vol. 25, no. 8, pp. 1859-1875, 2020.

[16] Karolinska Schizophrenia Project (KaSP) consortium, T. Elvsåshagen, S. Bahrami et al., "The genetic architecture of human brainstem structures and their involvement in common brain disorders," Nature Communications, vol. 11, no. 1, article 4016, 2020.

[17] S. Kim, S. Swaminathan, L. Shen et al., "Genome-wide association study of CSF biomarkers Abeta1-42, t-tau, and p-tau181p in the ADNI cohort," Neurology, vol. 76, no. 1, pp. 69-79, 2011.

[18] European Alzheimer's Disease Initiative (EADI), Genetic and Environmental Risk in Alzheimer's Disease (GERAD), Alzheimer's Disease Genetic Consortium (ADGC) et al., "Meta-analysis of 74,046 individuals identifies 11 new susceptibility loci for Alzheimer's disease," Nature Genetics, vol. 45, no. 12, pp. 1452-1458, 2013.

[19] R. D. Ball, "Designing a GWAS: power, sample size, and data structure," Methods in Molecular Biology, vol. 1019, pp. 3798, 2013

[20] G. Eraslan, Ž. Avsec, J. Gagneur, and F. J. Theis, "Deep learning: new computational modelling techniques for genomics," Nature Reviews Genetics, vol. 20, no. 7, pp. 389-403, 2019.

[21] B. Alipanahi, A. Delong, M. T. Weirauch, and B. J. Frey, "Predicting the sequence specificities of DNA- and RNA-binding proteins by deep learning," Nature Biotechnology, vol. 33, no. 8, pp. 831-838, 2015.

[22] J. Zhou and O. G. Troyanskaya, "Predicting effects of noncoding variants with deep learning-based sequence model," Nature Methods, vol. 12, no. 10, pp. 931-934, 2015.

[23] M. Wang, C. Tai, W. E, and L. Wei, "DeFine: deep convolutional neural networks accurately quantify intensities of transcription factor-DNA binding and facilitate evaluation of functional non-coding variants," Nucleic Acids Research, vol. 46, no. 11, article e69, 2018.

[24] H. Zeng and D. K. Gifford, "Predicting the impact of noncoding variants on DNA methylation," Nucleic Acids Research, vol. 45, no. 11, article e99, 2017.

[25] C. Angermueller, H. J. Lee, W. Reik, and O. Stegle, "DeepCpG: accurate prediction of single-cell DNA methylation states using deep learning," Genome Biology, vol. 18, no. 1, article 67, 2017.

[26] S. Cheng, M. Guo, C. Wang, X. Liu, Y. Liu, and X. Wu, "MiRTDL: a deep learning approach for miRNA target prediction," IEEE/ACM Transactions on Computational Biology and Bioinformatics, vol. 13, no. 6, pp. 1161-1169, 2016.

[27] J. Zhou, C. L. Theesfeld, K. Yao, K. M. Chen, A. K. Wong, and O. G. Troyanskaya, "Deep learning sequence-based ab initio prediction of variant effects on expression and disease risk," Nature Genetics, vol. 50, no. 8, pp. 1171-1179, 2018.

[28] R. Luo, F. J. Sedlazeck, T. W. Lam, and M. C. Schatz, “A multitask convolutional deep neural network for variant calling in single molecule sequencing," Nature Communications, vol. 10, no. 1, article 998, 2019.

[29] P. Chang, J. Grinband, B. D. Weinberg et al., "Deep-learning convolutional neural networks accurately classify genetic mutations in gliomas," AJNR. American Journal of Neuroradiology, vol. 39, no. 7, pp. 1201-1207, 2018.

[30] D. R. Kelley, Y. A. Reshef, M. Bileschi, D. Belanger, C. Y. McLean, and J. Snoek, "Sequential regulatory activity prediction across chromosomes with convolutional neural networks," Genome Research, vol. 28, no. 5, pp. 739-750, 2018.

[31] D. Quang and X. Xie, "FactorNet: a deep learning framework for predicting cell type specific transcription factor binding from nucleotide-resolution sequential data," Methods, vol. 166, pp. 40-47, 2019.

[32] G. M. McKhann, D. S. Knopman, H. Chertkow et al., "The diagnosis of dementia due to Alzheimer's disease: recommendations from the National Institute on Aging-Alzheimer's Association workgroups on diagnostic guidelines for Alzheimer's disease," Alzheimer's \& Dementia, vol. 7, no. 3, pp. 263-269, 2011.

[33] H. Neitzel, "A routine method for the establishment of permanent growing lymphoblastoid cell lines," Human Genetics, vol. 73, no. 4, pp. 320-326, 1986.

[34] L. Shen, S. Kim, S. L. Risacher et al., "Whole genome association study of brain-wide imaging phenotypes for identifying quantitative trait loci in MCI and AD: a study of the ADNI cohort," NeuroImage, vol. 53, no. 3, pp. 1051-1063, 2010.

[35] T. Han, C. Liu, W. Yang, and D. Jiang, "Deep transfer network with joint distribution adaptation: a new intelligent fault diagnosis framework for industry application," ISA Transactions, vol. 97, pp. 269-281, 2020.

[36] K. Chen, K. Chen, Q. Wang, Z. He, J. Hu, and J. He, "Shortterm load forecasting with deep residual networks," IEEE Transactions on Smart Grid, vol. 10, no. 4, pp. 3943-3952, 2019.

[37] D. Titov, J. Diehl-Schmid, K. Shi et al., "Metabolic connectivity for differential diagnosis of dementing disorders," Journal of Cerebral Blood Flow \& Metabolism, vol. 37, no. 1, pp. 252262, 2017.

[38] Alzheimer's Disease Neuroimaging Initiative, M. Wang, J. Jiang et al., "Individual brain metabolic connectome indicator based on Kullback-Leibler Divergence Similarity Estimation predicts progression from mild cognitive impairment to Alzheimer's dementia," European Journal of Nuclear Medicine and Molecular Imaging, vol. 47, no. 12, pp. 2753-2764, 2020.

[39] the European Alzheimer's Disease Initiative Investigators, J. C. Lambert, S. Heath et al., "Genome-wide association study identifies variants at CLU and CR1 associated with Alzheimer's disease," Nature Genetics, vol. 41, no. 10, pp. 10941099, 2009.

[40] R. Zhu, X. Liu, and Z. He, "Association between CLU gene rs11136000 polymorphism and Alzheimer's disease: an updated meta-analysis," Neurological Sciences, vol. 39, no. 4, pp. 679-689, 2018.

[41] W. Xu, L. Tan, and J. T. Yu, "The role of PICALM in Alzheimer's disease," Molecular Neurobiology, vol. 52, no. 1, pp. 399-413, 2015.

[42] Z. Wang, H. Lei, M. Zheng, Y. Li, Y. Cui, and F. Hao, "Metaanalysis of the association between Alzheimer disease and variants in GAB2, PICALM, and SORL1," Molecular Neurobiology, vol. 53, no. 9, pp. 6501-6510, 2016.

[43] I. M. Badamasi, M. S. Lye, N. Ibrahim, and J. Stanslas, "Genetic endophenotypes for insomnia of major depressive disorder and treatment-induced insomnia," Journal of Neural Transmission, vol. 126, no. 6, pp. 711-722, 2019. 
[44] A. Drago and D. de Ronchi, "HTR2A gene variants and psychiatric disorders: a review of current literature and selection of SNPs for future studies," Current Medicinal Chemistry, vol. 14, no. 19, pp. 2053-2069, 2007.

[45] X. Q. Wang, W. J. Huang, L. Su et al., "Neuroimaging advances regarding subjective cognitive decline in preclinical Alzheimer's disease," Molecular Neurodegeneration, vol. 15, no. 1, p. $55,2020$. 